\title{
Dynamics in Argumentation with Single Extensions: Abstraction Principles and the Grounded Extension
}

\author{
Guido Boella ${ }^{1}$ and Souhila $\mathrm{Kaci}^{2}$ and Leendert van der Torre ${ }^{3}$ \\ ${ }^{1}$ Department of Computer Science, University of Torino, Italy \\ guido@di.unito.it \\ ${ }^{2}$ Université Lille-Nord de France, Artois, CRIL, CNRS UMR 8188 - IUT de Lens, F-62307, \\ France \\ kaciecril.fr \\ ${ }^{3}$ Computer Science and Communication, University of Luxembourg, Luxembourg \\ leendert@vandertorre.com
}

\begin{abstract}
In this paper we consider the dynamics of abstract argumentation in Baroni and Giacomin's framework for the evaluation of extension based argumentation semantics. Following Baroni and Giacomin, we do not consider individual approaches, but we define general principles or postulates that individual approaches may satisfy. In particular, we define abstraction principles for the attack relation, and for the arguments in the framework. We illustrate the principles on the grounded extension. In this paper we consider only principles for the single extension case, and leave the multiple extension case to further research.
\end{abstract}

\section{Introduction}

Argumentation is a suitable framework for modeling interaction among agents. Dung introduced a framework for abstract argumentation with various kinds of so-called semantics. Baroni and Giacomin introduced a more general framework to study general principles of sets of semantics [1]. This is a very promising approach, since due to the increase of different semantics we need abstract principles to study the proposals, compare them, and select them for applications. So far Dung's argumentation framework has been mainly considered as static, in the sense that the argumentation framework is fixed. The dynamics of argumentation framework has attracted a recent interest where the problem of revising an argumentation framework has been addressed [5,7]. In this paper, we address complementary problems and study how the semantics of an argumentation framework remains unchanged when we change the set of arguments or the attack relations between them. In particular, we consider the case in which arguments or attack relations are removed, for example when agents retract arguments in a dialogue. More precisely, we address the following questions:

1. Which principles can be defined for abstracting (i.e., removing) an attack relation?

2. Which principles can be defined for abstracting (i.e., removing) an argument?

3. Which of these principles are satisfied by the grounded semantics?

We use the general framework of Baroni and Giacomin for arbitrary argumentation semantics, but we consider only semantics that give precisely one extension, like the 
grounded extension or the skeptical preferred semantics. Baroni and Giacomin [1] define the so-called directionality and resolution principles, which may be considered as argument and attack abstraction principles respectively. However, whereas directionality only considers abstraction from disconnected arguments, we also consider abstraction from arguments which are connected. To define the principles, we use Caminada's distinction between accepted, rejected and undecided arguments [4]. We find some results for the most popular semantics used in argumentation, namely the grounded extension.

In this paper we consider only principles for the single extension case, and leave the multiple extension case to further research. Moreover, we consider only abstractions which differ only one attack or one argument.

The layout of this paper is as follows. In Section 2 we give a recall of Dung's argumentation framework, the framework of Baroni and Giacomin, Caminada labeling, and we introduce the notion of abstraction. In Section 3 we consider the abstraction of attack relations and in Section 4 we consider the abstraction of arguments.

\section{Formal framework for abstraction principles}

\subsection{Dung's argumentation framework}

Argumentation is a reasoning model based on constructing arguments, determining potential conflicts between arguments and determining acceptable arguments. Dung's framework [6] is based on a binary attack relation. In Dung's framework, an argument is an abstract entity whose role is determined only by its relation to other arguments. Its structure and its origin are not known. We restrict ourselves to finite argumentation frameworks, i.e., those frameworks in which the set of arguments is finite.

Definition 1 (Argumentation framework). An argumentation framework is a tuple $\langle\mathcal{B}, \rightarrow\rangle$ where $\mathcal{B}$ is a finite set (of arguments) and $\rightarrow$ is a binary (attack) relation defined on $\mathcal{B} \times \mathcal{B}$.

The output of $\langle\mathcal{B}, \rightarrow\rangle$ is derived from the set of selected acceptable arguments, called extensions, with respect to some acceptability semantics. We need the following definitions before we recall the most widely used acceptability semantics of arguments given in the literature.

Definition 2. Let $\langle\mathcal{B}, \rightarrow\rangle$ be an argumentation framework. Let $\mathcal{S} \subseteq \mathcal{B}$.

- $\mathcal{S}$ defends a if $\forall b \in \mathcal{B}$ such that $b \rightarrow a, \exists c \in \mathcal{S}$ such that $c \rightarrow b$.

$-\mathcal{S} \subseteq \mathcal{B}$ is conflict-free if and only if there are no $a, b \in \mathcal{S}$ such that $a \rightarrow b$.

The following definition summarizes the well-known acceptability semantics.

Definition 3 (Acceptability semantics). Let $A F=\langle\mathcal{B}, \rightarrow\rangle$ be an argumentation framework. Let $\mathcal{S} \subseteq \mathcal{B}$.

$-\mathcal{S}$ is an admissible extension if and only if it is conflict-free and defends all its elements. 
- $\mathcal{S}$ is a complete extension if and only if it is conflict-free and $\mathcal{S}=\{a \mid \mathcal{S}$ defends a $\}$.

$-\mathcal{S}$ is a grounded extension of $A F$ if and only if $\mathcal{S}$ is the smallest (for set inclusion) complete extension of $A F$.

$-\mathcal{S}$ is a preferred extension of $A F$ if and only if $\mathcal{S}$ is maximal (for set inclusion) among admissible extensions of $A F$.

- $\mathcal{S}$ is the skeptical preferred extension of $A F$ if and only if $\mathcal{S}$ is the intersection of all preferred extensions of $A F$.

$-\mathcal{S}$ is a stable extension of $A F$ if and only if $\mathcal{S}$ is conflict-free and attacks all arguments of $\mathcal{B} \backslash \mathcal{S}$.

Which semantics is most appropriate in which circumstances depends on the application domain of the argumentation theory. The grounded extension is the most basic one, in the sense that its conclusions are not controversial, each argumentation framework has a grounded extension (it may be the empty set), and this extension is unique. The grounded extension therefore plays an important role in the remainder of this paper. The preferred semantics is more credulous than the grounded extension. There always exists at least one preferred extension but it does not have to be unique. Stable extensions have an intuitive appeal, but their drawbacks are that extensions do not have to be unique and do not have to exist. Stable extensions are used, for example, in answer set programming, where it makes sense that some programs do not have a solution.

\subsection{Baroni and Giacomin's framework}

In this paper we use the recently introduced formal framework for argumentation of Baroni and Giacomin [1]. They assume that the set $\mathcal{B}$ represents the set of arguments produced by a reasoner at a given instant of time, and they therefore assume that $\mathcal{B}$ is finite, independently of the fact that the underlying mechanism of argument generation admits the existence of infinite sets of arguments. Like in Dung's original framework, they consider an argumentation framework as a pair $\langle\mathcal{B}, \rightarrow\rangle$ where $\mathcal{B}$ is a set and $\rightarrow \subseteq(\mathcal{B} \times \mathcal{B})$ is a binary relation on $\mathcal{B}$, called the attack relation. In the following it will be useful to explicitly refer to the set of all arguments which can be generated, which we call $\mathcal{N}$ for the universe of arguments.

The generalization of Baroni and Giacomin is based on a function $\mathcal{E}$ that maps an argumentation framework $\langle\mathcal{B}, \rightarrow\rangle$ to its set of extensions, i.e., to a set of sets of arguments. However, this function is not formally defined. To be precise, they say: "An extensionbased argumentation semantics is defined by specifying the criteria for deriving, for a generic argumentation framework, a set of extensions, where each extension represents a set of arguments considered to be acceptable together. Given a generic argumentation semantics $\mathcal{S}$, the set of extensions prescribed by $\mathcal{S}$ for a given argumentation framework $A F$ is denoted as $\mathcal{E}_{\mathcal{S}}(A F)$." The following definition captures the above informal meaning of the function $\mathcal{E}$. Since Baroni and Giacomin do not give a name to the function $\mathcal{E}$, and it maps argumentation frameworks to the set of accepted arguments, we call $\mathcal{E}$ the acceptance function.

Definition 4. Let $\mathcal{N}$ be the universe of arguments. A multiple extensions acceptance function $\mathcal{E}: \mathcal{N} \times 2^{\mathcal{N} \times \mathcal{N}} \rightarrow 2^{2^{\mathcal{N}}}$ is 
1. a partial function which is defined for each argumentation framework $\langle\mathcal{B}, \rightarrow\rangle$ with finite $\mathcal{B} \subseteq \mathcal{N}$ and $\rightarrow \subseteq \mathcal{B} \times \mathcal{B}$, and

2. which maps an argumentation framework $\langle\mathcal{B}, \rightarrow\rangle$ to sets of subsets of $\mathcal{B}$ : $\mathcal{E}(\langle\mathcal{B}, \rightarrow\rangle) \subseteq 2^{\mathcal{B}}$.

The generality of the framework of Baroni and Giacomin follows from the fact that they have to define various principles which are built-in in Dung's framework. For example, Baroni and Giacomin identify the following two fundamental principles underlying the definition of extension-based semantics in Dung's framework, the language independent principle and the conflict free principle (see [1] for a discussion on these principles). In the following, we assume that these two principles are satisfied.

Definition 5 (Language independence). Two argumentation frameworks $\mathcal{A F}_{1}=\left\langle\mathcal{B}_{1}, \rightarrow_{1}\right\rangle$ and $\mathcal{A F}_{2}=\left\langle\mathcal{B}_{2}, \rightarrow_{2}\right\rangle$ are isomorphic if and only if there is a bijective mapping $m: \mathcal{B}_{1} \rightarrow \mathcal{B}_{2}$, such that $(\alpha, \beta) \in \rightarrow_{1}$ if and only if $(m(\alpha), m(\beta)) \in \rightarrow_{2}$. This is denoted as $\mathcal{A F}_{1} \doteq_{m} \mathcal{A F}_{2}$.

$A$ semantics $\mathcal{S}$ satisfies the language independence principle if and only if $\forall A F_{1}=\left\langle\mathcal{B}_{1}, \rightarrow_{1}\right\rangle, \forall A F_{2}=\left\langle\mathcal{B}_{2}, \rightarrow_{2}\right\rangle$ such that $A F_{1} \dot{\doteq}_{m} A F_{2}$ we have $\mathcal{E}_{\mathcal{S}}\left(A F_{2}\right)=$ $\left.\left\{M(E) \mid E \in \mathcal{E}_{\mathcal{S}}\left(A F_{1}\right)\right)\right\}$, where $M(E)=\left\{\beta \in \mathcal{B}_{2} \mid \exists \alpha \in E, \beta=m(\alpha)\right\}$.

Definition 6 (Conflict free). Given an argumentation framework $A F=\langle\mathcal{B}, \rightarrow\rangle$, a set $S \subseteq \mathcal{B}$ is conflict free, denoted as cf $(S)$, iff $\nexists a, b \in S$ such that $a \rightarrow b$. A semantics $\mathcal{S}$ satisfies the conflict free principle if and only if $\forall A F, \forall E \in \mathcal{E}_{\mathcal{S}}(A F), E$ is conflict free.

\subsection{The single extension case}

In this paper we consider only the case in which the semantics of an argumentation framework contains precisely one extension. Examples are the grounded and the skeptical preferred extension.

Definition 7. Let $\mathcal{N}$ be the universe of arguments. A single extension acceptance function $\mathcal{A}: \mathcal{N} \times 2^{\mathcal{N} \times \mathcal{N}} \rightarrow 2^{\mathcal{N}}$ is

1. a total function which is defined for each argumentation framework $\langle\mathcal{B}, \rightarrow\rangle$ with finite $\mathcal{B} \subseteq \mathcal{N}$ and $\rightarrow \subseteq \mathcal{B} \times \mathcal{B}$, and

2. which maps an argumentation framework $\langle\mathcal{B}, \rightarrow\rangle$ to a subset of $\mathcal{B}: \mathcal{A}(\langle\mathcal{B}, \rightarrow\rangle) \subseteq \mathcal{B}$.

Principles of Baroni and Giacomin defined for multiple acceptance functions such as directionality and conflict free are defined also for the single extension case, because the set of all single extension acceptance functions is a subset of the set of all multiple extensions acceptance functions. For example, a semantics $\mathcal{S}$ satisfies the conflict free principle when the unique extension is conflict free: $\forall A F, A_{\mathcal{S}}(A F)$ is conflict free.

\subsection{Abstraction}

We now define abstraction relations between argumentation frameworks.

Definition 8 (Abstraction). Let $\langle\mathcal{B}, \mathcal{R}\rangle$ and $\left\langle\mathcal{B}^{\prime}, \mathcal{S}\right\rangle$ be two argumentation frameworks. 
- $\langle\mathcal{B}, \mathcal{R}\rangle$ is an argument abstraction from $\left\langle\mathcal{B}^{\prime}, \mathcal{S}\right\rangle$ iff $\mathcal{B} \subseteq \mathcal{B}^{\prime}$ and $\forall a, b \in \mathcal{B}$, aR $b$ if and only if $a \mathcal{S} b$.

- $\langle\mathcal{B}, \mathcal{R}\rangle$ is an attack abstraction from $\left\langle\mathcal{B}^{\prime}, \mathcal{S}\right\rangle$ iff $\mathcal{B}=\mathcal{B}^{\prime}$ and $\mathcal{R} \subseteq \mathcal{S}$.

- $\langle\mathcal{B}, \mathcal{R}\rangle$ is an argument-attack abstraction from $\left\langle\mathcal{B}^{\prime}, \mathcal{S}\right\rangle$ iff $\mathcal{B} \subseteq \mathcal{B}^{\prime}$ and $\mathcal{R} \subseteq \mathcal{S}$.

Baroni and Giacomin also introduce two principles which may be interpreted as argument abstraction or attack abstraction principles, the so-called directionality and resolution principles. Directionality says that unattacked sets are unaffected by the remaining part of the argumentation framework as far as extensions are concerned (a principle which, as they show, does not hold for stable semantics, but it does hold for most other semantics). This may be seen as an argument abstraction principle, in the sense that when we abstract away all arguments not affecting a part of the argumentation framework, then the extensions of this part of the framework are not affected either (see their paper for the details).

\subsection{Caminada labeling}

In the definition of principles in the following section, it is useful to distinguish between rejected and undecided arguments. The following definition gives Caminada's [4] translation from extensions to three valued labelling functions. Caminada uses this translation only for complete extensions, such as the grounded extension, such that an argument is accepted if and only if all its attackers are rejected, and an argument is rejected if and only if it has at least one attacker that is accepted. We use it also for extensions which are not complete, such as the skeptical preferred extension, such that Caminada's labelling principles may not hold in general. We assume only that extensions are conflict free, i.e., an accepted argument cannot attack another accepted argument.

Definition 9 (Rejected and undecided arguments). Let $\mathcal{A}(A F)$ be a conflict free extension of an argumentation framework $A F=\langle\mathcal{B}, \rightarrow\rangle$, then $\mathcal{B}$ is partitioned into $\mathcal{A}(A F), \mathcal{R}(A F)$ and $\mathcal{U}(A F)$, where:

- $\mathcal{A}(A F)$ is the set of accepted arguments,

- $\mathcal{R}(A F)=\{a \in \mathcal{B} \mid \exists x \in \mathcal{A}(A F): x \rightarrow a\}$ is the set of rejected arguments, and

$-\mathcal{U}(A F)=\mathcal{B} \backslash(\mathcal{A}(A F) \cup \mathcal{R}(A F))$ is the set of undecided arguments.

\section{Attack abstraction principles}

In this section, we consider the situation where the set of arguments remains the same, but the attack relation may shrink (abstraction). Our framework considers principles where we remove a single attack relation $a \rightarrow b$ from an argumentation framework. We distinguish whether arguments $a$ and $b$ are accepted, rejected or undecided.

Principle 1 (Attack abstraction) An acceptance function $\mathcal{A}$ satisfies the $\mathcal{X} \mathcal{Y}$ attack abstraction principle, where $\mathcal{X}, \mathcal{Y} \in\{\mathcal{A}, \mathcal{R}, \mathcal{U}\}$, if for all argumentation frameworks $A F=\langle\mathcal{B}, \rightarrow\rangle, \forall a \in \mathcal{X}(A F) \forall b \in \mathcal{Y}(A F): \mathcal{A}(\langle\mathcal{B}, \rightarrow \backslash\{a \rightarrow b\}\rangle)=\mathcal{A}(A F)$. 
We start with two useful lemmas. The first says that there cannot be an attack relation from an accepted argument to another accepted or an undecided argument, denoted $\mathcal{A} \mathcal{A}$ and $\mathcal{A U}$ respectively.

Lemma 1. Each semantics that satisfies the consistency principle (and thus each semantics defined by Dung) satisfies the following principle.

$\mathcal{A A} ; \mathcal{A U}$ There is no attack from an accepted argument to an accepted or undecided argument.

Proof. (sketch) $\mathcal{A} \mathcal{A}$ is a reformulation of the consistency principle. $\mathcal{A U}$ follows directly from the notion of undecided in Definition 9.

The second lemma gives a new characterization of the distinction between grounded and skeptical preferred semantics. It says that there cannot be an attack relation from an undecided argument to an accepted argument, denoted $\mathcal{U} \mathcal{A}$. In other words, if an accepted argument is attacked, then its attacker is itself attacked by an accepted argument and thus rejected.

Lemma 2. The grounded semantics satisfies the following principle, whereas the skeptical preferred semantics does not.

$\mathcal{U} \mathcal{A}$ There is no attack from an undecided argument to an accepted argument.

Proof. (sketch) A counterexample for the skeptical preferred semantics is a well known example distinguishing the two semantics, that contains four arguments $\{a, b, c, d\}$ where $a$ and $b$ attack each other, both $a$ and $b$ attack $c$, and $c$ attacks $d$. The grounded extension is empty, whereas the skeptical preferred extension contains only d. All the other arguments are undecided, there are no rejected arguments. Thus, in the skeptical preferred semantics there is an undecided argument that attacks an accepted argument, whereas in the grounded semantics, there is no such argument. The fact that in the grounded semantics there are no undecided arguments attacking accepted arguments follows by structural induction on the construction of the grounded extension.

The following proposition shows that the grounded extension satisfies seven of the nine abstraction principles.

Proposition 1. The grounded semantics satisfies the $\mathcal{A} \mathcal{A}, \mathcal{A U}, \mathcal{U} \mathcal{A}, \mathcal{U} \mathcal{R}, \mathcal{R} \mathcal{A}, \mathcal{R U}$ and $\mathcal{R} \mathcal{R}$ attack abstraction principles, and it does not satisfy the $\mathcal{A R}$ and $\mathcal{U} \mathcal{U}$ attack abstraction principles. Intuitively the satisfied principles reflect the following ideas:

$\mathcal{A A} ; \mathcal{A U} ; \mathcal{U} \mathcal{A}$ hold vacuously, since there is nothing to remove (Lemma 1 and 2 ).

$\mathcal{R A} ; \mathcal{R} \mathcal{R} ; \mathcal{R U}:$ the attacks from a rejected argument do not influence the extension. This principle holds for any attacked argument $b$.

$\mathcal{U R}:$ the attacks on a rejected argument $b$ by an undecided argument a do not influence the extension. Intuitively, this means that an argument is rejected only when it is attacked by an accepted argument. 
Proof. (sketch) The satisfied principles can be proven by induction. Take the argumentation framework and the abstracted one, and show that in each step of the construction of the grounded extension, the two remain the same. Counterexamples for $\mathcal{U U}$ and $\mathcal{A R}$ attack abstraction are given below.

$\mathcal{U U}$ Consider an argumentation framework composed of two arguments a and b attacking each other so both will be undecided. If we remove the attack from a to $b$, then $b$ attacks $a$, so $b$ will be accepted and $a$ is rejected by the grounded extension, and all other reasonable acceptability semantics.

$\mathcal{A R}$ For the latter, consider again an argumentation framework composed of two arguments $a$ and $b$, where a attacks $b$. $a$ is accepted while $b$ is rejected. If we remove this attack relation, then both are accepted.

Proposition 1 leaves two interesting cases for further principles: the removal of an attack relation from an undecided argument to another undecided argument, i.e., $\mathcal{U} \mathcal{U}$, and the removal of an attack relation from an argument that is accepted to an argument that is rejected, i.e., $\mathcal{A R}$. In both cases, the extension can stay the same only under conditions: the challenge is therefore to define suitable conditions. We first define conditional attack abstraction. The idea is that if we remove an attack from $a$ to $b$, then in those two cases there must be another reason why $b$ does not become accepted.

Principle 2 (Conditional attack abstraction) An acceptance function $\mathcal{A}$ satisfies the $\mathcal{X} \mathcal{Y}(\mathcal{Z})$ attack abstraction principle, where $\mathcal{X}, \mathcal{Y}, \mathcal{Z} \in\{\mathcal{A}, \mathcal{R}, \mathcal{U}\}$, if for all argumentation frameworks $A F=\langle\mathcal{B}, \rightarrow\rangle$,

$$
\begin{gathered}
\forall a \in \mathcal{X}(A F) \forall b \mathcal{Y}(A F): \text { if } \exists c \in \mathcal{Z}(A F) \text { such that } c \neq a, c \rightarrow b \text { then } \\
\mathcal{A}(\langle\mathcal{B}, \rightarrow \backslash\{a \rightarrow b\}\rangle)=\mathcal{A}(A F),
\end{gathered}
$$

Proposition 2. The grounded semantics satisfies the $\mathcal{U} \mathcal{U}(\mathcal{A})$ and $\mathcal{U} \mathcal{U}(\mathcal{U})$ attack abstraction principles. It does not satisfy the $\mathcal{A R}(\mathcal{A}), \mathcal{A R}(\mathcal{U}), \mathcal{A R}(\mathcal{R}), \mathcal{U} \mathcal{U}(\mathcal{R})$ attack abstraction principles.

$\mathcal{U U}(\mathcal{A})$ holds vacuously since this situation never occurs.

$\mathcal{U} \mathcal{U}(\mathcal{U})$ represents that for attacks among undecided arguments, it is only important that there is at least one of such attacks, additional attacks to the same undecided argument do not change the extension.

Proof. (sketch) $\mathcal{U} \mathcal{U}(\mathcal{U})$ can be proven by induction. Take the argumentation framework and the abstracted one, and show that in each step of the construction of the grounded extension, the two remain the same.

$\mathcal{A R}(\mathcal{A})$ Consider three arguments $\{a, b, c\}$ with a attacks $b, b$ attacks $c$ and $c$ attacks $b$. The grounded extension is $\{a, c\}$, and $b$ is rejected. If we remove the attack from $a$ to $b$, then the grounded extension is $\{a\}$.

$\mathcal{A R}(\mathcal{U})$ Consider five arguments $\{a, b, c, d, e\}$ with a attacks $b, b$ attacks $c, c$ attacks $b, c$ attacks $d$ and $d$ attacks $c, b$ attacks $e$. The grounded extension is $\{a, e\}, b$ is rejected, $c$ and $d$ are undecided. If we remove the attack from a to $b$, then the grounded extension is $\{a\}$, all others are undecided. 
$\mathcal{A R}(\mathcal{R})$ Follows from Proposition 1. Due to $\mathcal{R} \mathcal{R}$ abstraction principle, we can remove the attack among the rejected arguments without changing the extension. Do this for all attacks among rejected arguments. Then due to the fact that grounded extension does not satisfy $\mathcal{A R}$, it also does not satisfy $\mathcal{A R}(\mathcal{R})$.

$\mathcal{U U}(\mathcal{R})$ Follows from Proposition 1. Due to $\mathcal{R} \mathcal{U}$ abstraction principle, we can remove the attack from the rejected arguments to the undecided arguments without changing the extension. Do this for all attacks among rejected arguments. Then due to the fact that grounded extension does not satisfy $\mathcal{U} \mathcal{U}$, it also does not satisfy $\mathcal{U} \mathcal{U}(\mathcal{R})$.

Note that the skeptical extension does not satisfy $\mathcal{U U}(\mathcal{U})$. Consider again the example given in the proof of Lemma 2. The preferred extensions are $\{a, d\}$ and $\{b, d\}$. So the skeptical extension is $\{d\}$. All other arguments are undecided. Let us now remove the attack from $a$ to $c$. Then the preferred extensions are $\{a, c\}$ and $\{b, d\}$. Thus the skeptical extension is empty. In other words, the $\mathcal{U U}(\mathcal{U})$ abstraction principle characterizes another distinction between the grounded and skeptical preferred semantics.

Summarizing, for argument $b$ to remain undecided when we remove the attack from $a$ to $b$, there must be another reason besides $a$ why $b$ is undecided. The other reason cannot be an accepted argument attacking $b$, since in that case $b$ would be rejected. Consequently the extension may change. And the other reason cannot be a rejected argument attacking $b$ since an attack from a rejected argument doesn't necessarily make an argument undecided. Therefore, we ask that there is another undecided argument $c$ attacking $b$. This motivates the $\mathcal{U} \mathcal{U}(\mathcal{U})$ principle.

Proposition 2 leaves two interesting cases for further development, $\mathcal{A R}(\mathcal{A})$ and $\mathcal{A R}(\mathcal{U})$ abstraction. We start with $\mathcal{A R}(\mathcal{A})$. Due to the removal of the attack, the status of argument $b$ may remain rejected or change from rejected to accepted or undecided. When the argument becomes accepted then it should belong to the extension following all reasonable acceptability semantics. Therefore we consider the cases where $b$ remains rejected or becomes undecided. When $b$ is still rejected, this means that there is another accepted argument $c$ unequal to $a$, which attacks $b$. This motivates $\mathcal{A R}(\mathcal{A})$ principle. However, the counterexample shows that the reason that $c$ is accepted, may be $b$ itself! The counterexample indicates ways in which conditional abstraction can be further developed: the rejection of $b$ should not be the reason for the acceptance of $c$.

One simple way to prevent the possibility that the rejection of $b$ is the cause of the acceptance of $c$, is to prevent any paths from $b$ to $c$. In fact, we can do a little better, because only odd paths can lead to acceptance. This motivates the following principle.

Principle 3 (Acyclic conditional attack abstraction) An acceptance function $\mathcal{A}$ satisfies the acyclic $\mathcal{X} \mathcal{Y}(\mathcal{Z})$ attack abstraction principle, where $\mathcal{X}, \mathcal{Y}, \mathcal{Z} \in\{\mathcal{A}, \mathcal{R}, \mathcal{U}\}$, if for all argumentation frameworks $A F=\langle\mathcal{B}, \rightarrow\rangle$,

$\forall a \in \mathcal{X}(A F) \forall b \mathcal{Y}(A F):$ if $\exists c \in \mathcal{Z}(A F)$ such that $c \neq a, c \rightarrow b$, and there is no odd length sequence of attacks from $b$ to $c$, then $\mathcal{A}(\langle\mathcal{B}, \rightarrow \backslash\{a \rightarrow b\}\rangle)=\mathcal{A}(A F)$,

Proposition 3. The grounded semantics satisfies the acyclic $\mathcal{A R}(\mathcal{A})$ abstraction principle.

We now consider $\mathcal{A R}(\mathcal{U})$. Thus there is an accepted argument $a$ attacking rejected argument $b$, which is also attacked by undecided argument $c$. In this case, the argument 
$b$ may change into undecided. In that case, argument $b$ is still not in the extension, but there may be implications in other parts of the argumentation framework. As the counterexample in Proposition 2 shows, $b$ should not be the cause of acceptance of another argument.

Principle 4 (Stronger conditional attack abstraction) An acceptance function $\mathcal{A}$ satisfies the $\mathcal{X} \mathcal{Y}(\mathcal{Z}, \mathcal{W})$ attack abstraction principle, if for all argumentation frameworks $A F=\langle\mathcal{B}, \rightarrow\rangle$,

$$
\begin{gathered}
\forall a \in \mathcal{X}(A F) \forall b \mathcal{Y}(A F): \text { if } \exists c \in \mathcal{Z}(A F) \text { such that } c \neq a, c \rightarrow b \text { and } \forall d \in \mathcal{W}(A F), \\
\text { we do not have } b \rightarrow d \text {, then } \mathcal{A}(\langle\mathcal{B}, \rightarrow \backslash\{a \rightarrow b\}\rangle)=\mathcal{A}(A F),
\end{gathered}
$$

where $\mathcal{X}, \mathcal{Y}, \mathcal{Z}, \mathcal{W} \in\{\mathcal{A}, \mathcal{R}, \mathcal{U}\}$.

Proposition 4. The grounded semantics satisfies the $\mathcal{A R}(\mathcal{U}, \mathcal{A})$ abstraction principle.

\section{Argument abstraction principles}

In this section we consider the abstraction of an argument, including the attack relations involving this argument. It builds on the abstraction principles for attack relations in the previous section. As before, we distinguish between removal of an argument which is accepted, which is rejected and which is undecided. In the first case the extension should be the extension without the accepted argument, in the other two cases the extension should stay the same. So we have to consider three cases. Let $\rightarrow_{a}$ denote the set of attack relations related to an argument $a$. Formally, we have the following principles:

Principle 5 (Argument abstraction) An acceptance function $\mathcal{A}$ satisfies the $\mathcal{X} \in\{\mathcal{R}, \mathcal{U}, A\}$ argument abstraction principle if for all argumentation frameworks $A F=\langle\mathcal{B}, \rightarrow\rangle$, if $a \in \mathcal{X}(A F)$, then $\mathcal{A}\left(\left\langle\mathcal{B} \backslash\{a\}, \rightarrow \backslash \rightarrow_{a}\right\rangle\right)=\mathcal{A}(A F) \backslash\{a\}$.

The following proposition shows that only $\mathcal{R}$ abstraction is satisfied. For example, if we remove an accepted argument, then arguments attacked by the removed accepted argument may become accepted.

Proposition 5. The grounded extension satisfies $\mathcal{R}$ argument abstraction, and it does not satisfy $\mathcal{A}$ and $U$ argument abstraction. This represents the following idea.

$\mathcal{R}$ Rejected arguments do not play a role in the argumentation and can be removed.

Proof. (sketch) Follows from the abstraction principles of the attack relation or their counterexamples in Proposition 1. For $\mathcal{U}$ abstraction take two arguments a and b attacking each other, and for $\mathcal{A}$ abstraction take two arguments $a$ and $b$ where $a$ attacks $b$.

This leave two interesting cases, $\mathcal{U}$ abstraction and $\mathcal{A}$ abstraction. We start with $\mathcal{U}$ abstraction. Unlike rejected arguments whose attacks are inoffensive, an attack from an undecided argment may "block" an argument, i.e., prevents the argument to be accepted. Therefore the removal of an undecided argument may change the extension. One way to keep the extension unchanged is that the removed undecided argument only attacks arguments which are in the extension (so the attacks have not been successful) or out of the extension but due to other arguments. Formally, we have the following principles: 
Principle $6(\mathcal{U}$ argument abstraction 1) $\mathcal{A}$ satisfies the undecided argument abstraction 1 principle if for all argumentation frameworks $A F=\langle\mathcal{B}, \rightarrow\rangle$, if a $\in \mathcal{U}(A F)$ is an undecided argument and a attacks only accepted arguments in $\mathcal{A}(A F)$, then $\mathcal{A}\left(\left\langle\mathcal{B} \backslash\{a\}, \rightarrow \backslash \rightarrow_{a}\right\rangle\right)=\mathcal{A}(A F)$.

Principle $7(\mathcal{U}$ argument abstraction 2) $\mathcal{A}$ satisfies the undecided argument abstraction 2 principle if for all argumentation frameworks $A F=\langle\mathcal{B}, \rightarrow\rangle$, if $a \in \mathcal{U}(A F)$ is an undecided argument and a attacks only rejected arguments in $\mathcal{R}(A F)$, then $\mathcal{A}\left(\left\langle\mathcal{B} \backslash\{a\}, \rightarrow \backslash \rightarrow_{a}\right\rangle\right)=\mathcal{A}(A F)$.

Moreover, inspired by $\mathcal{U} \mathcal{U}(\mathcal{U})$ attack abstraction principle, we define the following $\mathcal{U}$ argument abstraction principle.

Principle $8(\mathcal{U}$ argument abstraction 3) $\mathcal{A}$ satisfies the undecided argument abstraction 3 principle if for all argumentation frameworks $A F=\langle\mathcal{B}, \rightarrow\rangle$, if a $\in \mathcal{U}(A F)$ is an undecided argument and for each undecided argument $b$ attacked by $a$, there is another undecided argument $c \neq b$ that attacks $b$, then $\mathcal{A}\left(\left\langle\mathcal{B} \backslash\{a\}, \rightarrow \backslash \rightarrow_{a}\right\rangle\right)=\mathcal{A}(A F)$.

Proposition 6. The grounded extension satisfies $\mathcal{U}$ argument abstraction principle 1, 2 and 3.

Proof. (sketch) $\mathcal{U}$ argument abstraction 1 principle holds vacuously (Lemma 2). The others follow from Proposition 1 and 2.

Finally, we consider weakened $\mathcal{A}$ abstraction principles. The first principle of accepted argument abstraction is Baroni and Giacomin's principle of directionality restricted to single arguments. If we remove an argument that does not attack another argument besides possibly itself, then the extensions will remain the same.

Principle 9 ( $\mathcal{A}$ abstraction 1) $\mathcal{A}$ satisfies the accepted argument abstraction 1 principle if for all argumentation frameworks $A F=\langle\mathcal{B}, \rightarrow\rangle$, if $a \in \mathcal{A}(A F)$ is an accepted argument, and there is no argument $b \in \mathcal{B}$ unequal to a such that a attacks $b$, then $\mathcal{A}\left(\left\langle\mathcal{B} \backslash\{a\}, \rightarrow \backslash \rightarrow_{a}\right\rangle\right)=\mathcal{A}(A F) \backslash\{a\}$.

The second principle of accepted argument abstraction is inspired by Proposition 3. It says that if we remove an argument $b \in \mathcal{B}$ which attacks only arguments which are also attacked by other arguments, then the extensions remain the same.

Principle $10(\mathcal{A}$ abstraction 2) $\mathcal{A}$ satisfies the accepted argument abstraction 2 principle if for all argumentation frameworks $A F=\langle\mathcal{B}, \rightarrow\rangle$, if $a \in \mathcal{A}(A F)$ is an accepted argument, and for each rejected argument $b \in \mathcal{R}(A F)$ such that $a \rightarrow b$, there is another argument $c \in \mathcal{A}(A F)$ such that $c \rightarrow b$, and there is no odd attack sequence from $b$ to $c$, then $\mathcal{A}\left(\left\langle\mathcal{B} \backslash\{a\}, \rightarrow \backslash \rightarrow a_{a}\right\rangle\right)=\mathcal{A}(A F) \backslash\{a\}$

Proposition 7. The grounded extension satisfies $\mathcal{A}$ abstraction 1 and 2 principles. 


\section{Related research}

Besides the work of Baroni and Giacomin on principles for the evaluation of argumentation semantics, there is various work on dialogue and a few very recent approaches on the dynamics of argumentation. Researchers in the multi-agent systems area have been looking at this problem under various names. Cayrol et al. [5] define a typology of refinement (the dual of abstraction) (called revision in their paper), i.e. adding an argument. Then they define principles and condition so that each type of refinement becomes a revision (called classical revision in their paper), i.e., the new argument is accepted. Refinement and revision is different from abstraction considered in this paper, and they do not define general principles as we do. Rotstein et al. [7] introduce the notion of dynamics into the concept of abstract argumentation frameworks, by considering arguments built from evidence and claims. They do not consider abstract arguments and general principles as we do in this paper. Barringer et al. [2] consider internal dynamics by extending Dung's theory in various ways, but without considering general principles.

\section{Summary and further research}

Motivated by situations where the argumentation framework may change, for example by dialogues in which arguments can be retracted or added, we study principles where the extension does not change when we consider the dynamics of the argumentation framework. The most interesting attack abstraction principles are listed in Table 1. This table should be read as follows. Each line represents a principle. For example, the first line says that $\mathcal{A}$ satisfies the $\mathcal{R} \mathcal{A} ; \mathcal{U} \mathcal{A}$ principle if for all argumentation frameworks $A F=\langle\mathcal{B}, \rightarrow\rangle$, if $b \in \mathcal{A}(A F)$ is an accepted argument, then for all arguments $a \in \mathcal{R}(A F) \cup \mathcal{U}(A F), \mathcal{A}(\langle\mathcal{B}, \rightarrow \backslash\{a \rightarrow b\}\rangle)=\mathcal{A}(A F)$. For each principle, we state whether it is satisfied by the grounded extension or not. Vacuously means that the situation does not occur for the grounded extension.

\begin{tabular}{|c|c|c|}
\hline Principle & Condition & Grounded extension \\
\hline $\mathcal{A} \mathcal{A}, \mathcal{A U}, \mathcal{U} \mathcal{A}$ & $\begin{array}{c}a \in \mathcal{A}(A F) \cup \mathcal{U}(A F), b \in \mathcal{A}(A F) \text { or } \\
a \in \mathcal{A}(A F), b \in \mathcal{A}(A F) \cup \mathcal{U}(A F)\end{array}$ & yes (vacuously) \\
\hline $\mathcal{R} \mathcal{A}, \mathcal{R} \mathcal{R}, \mathcal{R U}$ & $a \in \mathcal{R}(A F)$ & yes \\
\hline $\mathcal{U R}$ & $a \in \mathcal{U}(A F), b \in \mathcal{R}(A F)$ & yes \\
\hline $\mathcal{U} \mathcal{U}(\mathcal{A})$ & $a \in \mathcal{U}(A F), b \in \mathcal{U}(A F), \exists c \in \mathcal{A}(A F), c \rightarrow b$ & yes (vacuously) \\
\hline $\mathcal{U} \mathcal{U}(\mathcal{U})$ & $a \in \mathcal{U}(A F), b \in \mathcal{U}(A F), \exists c \in \mathcal{U}(A F), c \neq a, c \rightarrow b$ & yes \\
\hline $\begin{array}{c}\mathcal{A R}(\mathcal{U}), \mathcal{A R}(\mathcal{R}) \\
\mathcal{U U}(\mathcal{R})\end{array}$ & & no \\
\hline $\mathcal{A R}(\mathcal{A})$ & $\begin{array}{c}a \in \mathcal{A}(A F), b \in \mathcal{R}(A F), \exists c \in \mathcal{A}(A F), c \neq a, c \rightarrow b \\
\text { no odd length sequence of attacks from } b \text { to } c\end{array}$ & yes \\
\hline $\mathcal{A R}(\mathcal{U}, \mathcal{A})$ & $\begin{array}{c}a \in \mathcal{A}(A F), b \in \mathcal{R}(A F), \exists c \in \mathcal{U}(A F), c \rightarrow b \\
\forall d \in \mathcal{A}(A F), \text { we do not have } b \rightarrow d\end{array}$ & yes \\
\hline
\end{tabular}

Table 1. Attack abstraction: if $\forall A F=\langle\mathcal{B}, \rightarrow\rangle$ condition, then $\mathcal{A}(\langle\mathcal{B}, \rightarrow \backslash\{a \rightarrow b\}\rangle)=\mathcal{A}(A F)$ 
Moreover, the most interesting argument abstraction principles are listed in Table 2.

\begin{tabular}{c|c|c} 
Principle & Condition & Grounded extension \\
\hline $\mathcal{R}$ & $a \in \mathcal{R}(A F)$ & yes \\
$\mathcal{U}$ & $a \in \mathcal{U}(A F)$ & no \\
$\mathcal{U} 1$ & $a \in \mathcal{U}(A F)$, if $a \rightarrow c$ then $c \in \mathcal{A}(A F)$ & yes (vacuously) \\
$\mathcal{U} 2$ & $a \in \mathcal{U}(A F)$, if $a \rightarrow c$ then $c \in \mathcal{R}(A F)$ & yes \\
$\mathcal{U} 3$ & $a \in \mathcal{U}(A F)$, if $a \rightarrow c$ and $c \in \mathcal{U}(A F)$ then $\ldots$ & yes \\
$\mathcal{A}$ & $a \in \mathcal{A}(A F)$ & no \\
$\mathcal{A} 1$ & $a \in \mathcal{A}(A F), \nexists b \neq a, a \rightarrow b$ & yes \\
$\mathcal{A} 2$ & $a \in \mathcal{A}(A F), \forall b \in \mathcal{R}(A F), a \rightarrow b, \exists c \in \mathcal{A}(A F), c \rightarrow b$ & yes
\end{tabular}

Table 2. Argument abstraction: if $\forall A F=\langle\mathcal{B}, \rightarrow\rangle$ condition, then $\mathcal{A}\left(\left\langle\mathcal{B} \backslash\{a\}, \rightarrow \backslash \rightarrow_{a}\right\rangle\right)=$ $\mathcal{A}(A F) \backslash\{a\}$.

There are several directions of future research. We would like to extend our dynamic analysis to a wider range of principles. For example, we are interested in refinement of argumentation frameworks [3], and their relation to argument abstraction. Moreover, besides considering situations in which the extension stays the same, we are interested in questions about the change needed to change an argument from being accepted to rejected, or vice versa. Also, we would like to study principles for the multiple extension case. Also, we would like to apply our theory to practical problems such as evaluation of argumentation semantics, or sensitivity analysis of a dispute.

\section{References}

1. P. Baroni and M. Giacomin. On principle-based evaluation of extension-based argumentation semantics. Artificial Intelligence, 171(10-15):675-700, 2007.

2. H. Barringer, D.M. Gabbay, and J. Woods. Temporal dynamics of support and attack networks: From argumentation to zoology. In Dieter Hutter and Werner Stephan, editors, Mechanizing Mathematical Reasoning, Essays in Honor of Jrg H. Siekmann on the Occasion of His 60th Birthday, Lecture Notes in Computer Science 2605, pages 59-98. Springer, 2005.

3. G. Boella, S. Kaci, and L. van der Torre. Dynamics in argumentation with single extensions: Attack refinement and the grounded extension (short paper). In Proc. of 8th Int. Conf. on Autonomous Agents and Multiagent Systems (AAMAS 2009), 2009.

4. M. Caminada. On the issue of reinstatement in argumentation. In 10th European Conference on Logics in Artificial Intelligence (JELIA'06), pages 111-123, 2006.

5. C. Cayrol, F. Dupin de Saint Cyr Bannay, and M.C. Lagasquie-Schiex. Revision of an argumentation system. In 11th International Conference on Principles of Knowledge Representation and Reasoning (KR'08), pages 124-134, 2008.

6. P.M. Dung. On the acceptability of arguments and its fundamental role in nonmonotonic reasoning, logic programming and n-person games. Artificial Intelligence, 77(2):321-357, 1995.

7. N. D. Rotstein, M. O. Moguillansky, A. J. Garcia, and G. R. Simari. An abstract argumentation framework for handling dynamics. In Proceedings of the Argument, Dialogue and Decision Workshop in NMR 2008, pages 131-139, Sydney, Australia, 2008. 